\title{
Characterization of important odorants in four steamed Coilia ectenes from China by gas chromatography-mass spectrometry-olfactometry
}

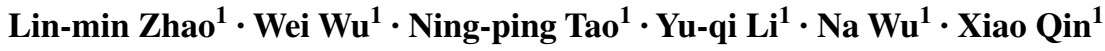

Received: 9 April 2015 / Accepted: 17 June 2015 / Published online: 28 July 2015

(C) The Author(s) 2015. This article is published with open access at Springerlink.com

\begin{abstract}
Odorants were extracted from four Coilia ectenes (Yangtze River-Coilia, East China sea-Coilia, Chao Lake-Coilia and Yellow River-Coilia) by MonoTrap ${ }^{\mathrm{TM}}$ and analyzed by the headspace-monolithic material sorptive extraction technique combined with gas chromatography-mass spectrometry-olfactometry (GC-MS-O). A total of 63 volatile compounds were identified. The results of GC-MS-O analysis associated with odor intensity revealed that trimethylamine (fishy, ammonia-like), 1-penten-3-ol (mushroom, cabbage), $N, N$-diethyl-formamide (roasted meaty), hexanal (grassy, earthy), ethylbenzene (nutty, floral), (Z)-4-heptenal (fishy, boiled potato), benzaldehyde (almond, metallic), 1-octen-3-ol (mushroom, cabbage), nonanal (oily), and decanal (green, oily) were the important odorants in the four species of Coilia ectenes. Furthermore, trimethylamine and 1-penten-3-ol were common to four Coilia ectenes. TMA (fishy, ammonia-like), 1-penten-3-ol (mushroom, cabbage), N,N-diethyl-formamide (roasted meaty), hexanal (grassy, earthy), (Z)-4-heptenal (fishy, boiled potato), and benzaldehyde (almond, metallic) were included in Yangtze River-Coilia; TMA, 1-penten-3-ol, ethylbenzene (nutty, floral), (Z)-4-heptenal, 1-octen-3-ol (mushroom, earthy), and nonanal (oily) in East China seaCoilia; TMA, 1-penten-3-ol, ethylbenzene, 1-octen-3-ol and nonanal in Chao Lake-Coilia; and TMA, 1-penten-3-ol,
\end{abstract}

Ning-ping Tao

nptao@shou.edu.cn

Lin-min Zhao

2767620467@qq.com

1 College of Food Science and Technology, Shanghai Ocean University, No. 999, Hucheng Huan Rd, Lingang New City, 201306 Shanghai, China hexanal, (Z)-4-heptenal, 1-octen-3-ol and decanal (green, oily) in Yellow River-Coilia.

Keywords Coilia ectenes $\cdot$ Headspace-monolithic material sorptive extraction $\cdot$ GC-MS-O $\cdot$ MonoTrap $^{\mathrm{TM}}$. Important odorants

\section{Introduction}

Coilia ectenes related to Osteichthyes, Clupeiformes, and Engraulidae, is an important fish species that migrates from near-ocean waters to freshwater rivers every spring. In China, Coilia ectenes is mainly classified into the Jianghai migratory type and Lake settlement type according to its survival mode. The Jianghai migratory type migrates periodically between river and sea for some reason, which includes physiological requirements, genetic, and environment factors. The Jianghai migratory type includes Yangtze River-Coilia and East China sea-Coilia. The lake settlement type is a kind of Coilia ectenes living in lakes all through its lifetime, including Chao Lake-Coilia and Yellow RiverCoilia [1]. It was reported that some Coilia ectenes species do not migrate to freshwater rivers due to genetic or environment factors, so there are significant differences between the river-anchovy and sea-anchovy [2]. Coilia ectenes has a high economic value for its distinctive aroma, intensive umami taste, and high nutritional value. However, its declining production fails to meet the market demands due to overfishing and environmental pollution, thereby contributing to the rise in prices (Yangtze River-Coilia is $8000-12000$ yuan/kg, i.e., approximately 1333-2000 US\$/ $\mathrm{kg}$ ) and to the development of Coilia ectenes farming.

MonoTrap $^{\mathrm{TM}}$ (MT) is a novel absorbent composed of silica gel, activated carbon, and octadecyl silane (ODS). 
It is widely used in the extraction of polar and non-polar compounds as well as those with high and low boiling points. When the samples pass through the pores of the silica monolithic structure, they are trapped by the ODS groups, which are bound to the silica surface or the activated carbon present inside and outside the structure. This mode of absorption is called monolithic material sorptive extraction (MMSE), which is similar to solid-phase micro extraction (SPME) and stir bar sorptive extraction (SBSE). The headspace MMSE (HS-MMSE) with a larger specific surface area and porous surface [3], has higher adsorption efficiency than the headspace SPME (HS-SPME) and the headspace SBSE (HS-SBSE). HS-MMSE has been applied to plants, coffee, tea, sesame oil, and dairy products [4].

The GC-MS-O technology, which was first proposed by Fuller in 1964, is a useful method for evaluation of odorants from complex materials, including the frequency detection method, the dilution method and the direct intensity method [5]. The frequency detection method was used to detect the odor frequency by assessors and described by Linssen in 1993 [6]. The dilution method is the dilution level at which flavor compounds cannot be smelled by the human nose [7]. The direct intensity method could offer several advantages of reducing sniffing time and errors by recording the changes of odor intensities directly, which is superior to frequency detection and the dilution method [8]. Several researchers have analyzed odor-active compounds in seafoods by GC-MS-O, such as the New Zealand sea urchin Evechinus chloroticus [9], rainbow trout Oncorhynchus mykiss [10], and Yangtze River-Coilia [11]. However, few works have focused on the study of flavor compounds in Coilia ectenes by GC-MS-O associated with odor intensity.

The objective of this study was to identify the important odorants in steamed Yangtze River-Coilia, East China sea-Coilia, Chao Lake-Coilia and Yellow River-Coilia by GC-MS-O (direct intensity method) as associated with the odor intensity method. This study may provide a theoretical basis for further research and development in breeding technologies of Coilia ectenes or other aquatic products.

\section{Materials and methods}

\section{Materials}

Ten Yangtze River-Coilia (average weight and length $109.5 \pm 7.7 \mathrm{~g}$ and $26.3 \pm 0.6 \mathrm{~cm}$, respectively) and ten East China sea-Coilia (average weight and length $106.1 \pm 4.5 \mathrm{~g}$ and $25.9 \pm 1.1 \mathrm{~cm}$, respectively) were purchased from the Xiaohu Aquatic Products Market (Jiangsu, China) in April 2012; ten Chao Lake-Coilia (average weight and length $25.2 \pm 9.4 \mathrm{~g}$ and $20.4 \pm 2.0 \mathrm{~cm}$, respectively) were purchased from Fuhuang Sungem Food Company (Anhui, China) in October 2012; and ten Yellow River-Coilia (average weight and length $91.5 \pm 6.4 \mathrm{~g}$ and $29.9 \pm 1.7 \mathrm{~cm}$, respectively) were purchased from Dongying Jingming Aquatic Products Company (Shandong, China) in April 2012.

All specimens were transported to the laboratory with dry ice, fishbone and liver were removed, and then stored at $-80{ }^{\circ} \mathrm{C}$ until required for analysis.

$\mathrm{C}_{6}-\mathrm{C}_{30} \quad n$-alkanes standards were purchased from Sigma-Aldrich Trading Co., Ltd (Shanghai, China). Other reagents used were of analytical grade and purchased from Anpel Laboratory Technologies Co., Ltd (Shanghai, China).

\section{HS-MMSE for volatile compounds extraction}

Yangtze River-Coilia, East China sea-Coilia, Chao LakeCoilia, and Yellow River-Coilia were rinsed under running water, eviscerated, steamed for $20 \mathrm{~min}$ to guarantee the Coilia ectenes was fully cooked, and minced. After cooling to room temperature, the Coilia ectenes were homogenized in an ice-bath condition (Model JZ-II, Tianjin Sifang Equipment Ltd, China). Prior to use, MonoTrap ${ }^{\mathrm{TM}}$ rods were conditioned in an oven at $250{ }^{\circ} \mathrm{C}$ for $30 \mathrm{~min}$ to remove any impurities and inserted into the stainless steel MT holders using a set of clean tweezers to avoid contamination, and the stainless steel MT holder was inserted into the MT stand. Nine MT rods were applied in this study for achieving the greatest extraction of volatile compounds in Coilia ectenes. A clean septum was passed through the end of the MT holder using clean tweezers, and a cap was placed on the top of the holder. MT rods were placed at a fixed position in the headspace of a $15 \mathrm{~mL}$-vial (item Nr. VAAP38015E-1760A-100, Shanghai Anpu Scientific Instrument Co., Ltd.) containing approximately $5.0 \mathrm{~g}$ homogenized sample, and a septum was hermetically sealed on the vial, which was kept in a heat-gathering style magnetism mixer for $60 \mathrm{~min}$ at $80^{\circ} \mathrm{C}$. Volatile compounds were exposed and adsorbed to both side surfaces of the MT rods. Following adsorption, the MT rods were removed and immediately placed carefully in an adsorption tube, then desorbed via a thermal desorption unit (TDU, Gerstel, Baltimore,Md., USA) and injected via a cold injection system (CIS, Gerstel, Baltimore,Md., USA). The TDU temperature was programmed at $180{ }^{\circ} \mathrm{C} / \mathrm{min}$ from 50 to $270{ }^{\circ} \mathrm{C}$ and maintained for $5 \mathrm{~min}$; the CIS temperature was programmed at $12{ }^{\circ} \mathrm{C} / \mathrm{s}$ from -40 to $250{ }^{\circ} \mathrm{C}$ and maintained for $0.5 \mathrm{~min}$.

\section{Gas chromatography-mass spectrometry-olfactometry (GC-MS-O)}

A gas chromatography-mass spectrometer (Model 6980, Agilent Inc., USA) was coupled to an ODP 2 sniffing 
port (Gerstal). The effluent from the capillary column was split $(1: 1.5 \mathrm{v} / \mathrm{v})$ between the mass spectrometry detector (MSD) and the ODP-2. Separations in the GC were performed on a DB-5MS capillary column $(60 \mathrm{~m}$ length $\times 0.25 \mathrm{~mm}$ internal diameter $\times 0.25 \mu \mathrm{m}$ film thickness; Agilent Inc., USA), using helium (99.999\% space purity) as the carrier gas $(1.2 \mathrm{~mL} / \mathrm{min})$. The oven temperature was programmed from 40 to $100{ }^{\circ} \mathrm{C}$ at a rate of $5{ }^{\circ} \mathrm{C} / \mathrm{min}$, then increased to $180{ }^{\circ} \mathrm{C}$ at a rate of $2{ }^{\circ} \mathrm{C} /$ $\mathrm{min}$, followed by an increase at $5{ }^{\circ} \mathrm{C} / \mathrm{min}$ to $240{ }^{\circ} \mathrm{C}$ and maintained for $5 \mathrm{~min}$.

MS conditions were as follows: detector interface temperature was $250{ }^{\circ} \mathrm{C}$, ion source temperature was $230{ }^{\circ} \mathrm{C}$, ionization energy was $70 \mathrm{Ev}$, mass range was $40-450$ amu, electron multiplier voltage was $1576 \mathrm{~V}$, and scan rate was $1.8 / \mathrm{s}$. Samples were desorbed at $270{ }^{\circ} \mathrm{C}$ with a TDU (Gerstal) directly into the hot injector $\left(250{ }^{\circ} \mathrm{C}\right)$ of the CIS (Gerstal) with simultaneous cryofocusing using liquid nitrogen.

In the GC-MS-O study, ten experienced assessors with previous sniffing experience (five male and five females, 22-30 years of age) were choose as candidates in the GC-MS-O panelist selection. Based on the guidelines established by Pollien and others [12], three assessors (one male and two females) were required to take part in GC-MS-O detection due to their high sensitive olfaction and recorded the aroma characteristics and aroma strength of each sample. Each sample was smelled twice by the assessors. The mean of odor intensity was calculated and the final odor intensities were obtained from the mean values of at least two assessors. During a 50-min sniffing period per sample, assessors ranked the intensity of each odor on a four-point intensity scale: $1=$ weak, $2=$ moderate, $3=$ strong, and $4=$ very strong [8].

\section{Statistical analyses}

All detections had been replicated three times in this study. Results were expressed as mean \pm standard deviation (SD) $(n=3)$. The SPSS 19.0 software (SPSS Inc., Chicago, III., USA) was applied to check if there were significant differences $(p<0.05)$ between four Coilia ectenes based on oneway analysis of variance (ANOVA).

Volatile compounds were analyzed by matching the mass spectra (Wiley/NIST 2008) and the linear retention index (LRI) with reference values and odor properties. The LRI was calculated by the following equation,

$L R I=\left(\frac{R t_{(x)}-R t_{(n)}}{R t_{(n+1)}-R t_{(n)}}+n\right) \times 100$,

where $\operatorname{Rt}(\chi)$ is the retention time of each volatile compound $(\chi), \operatorname{Rt}(n)$ and $\operatorname{Rt}(n+1)$ are the retention times of $n$-alkanes eluting directly before and after the compound $(x)$ under identical chromatographic conditions.

\section{Results}

The total ion chromatogram obtained from Coilia ectenes samples is shown in Fig. 1. A total of 63 volatile compounds were identified in steamed meat of Yangtze RiverCoilia, East China sea-Coilia, Chao Lake-Coilia, and Yellow River-Coilia on a DB-5ms column (Table 1): including 19 aldehydes, 11 ketones, 11 alcohols, 8 aromatics, 5 hydrocarbons, $6 \mathrm{~N}$-and $S$ - containing compounds, and 3 furans. Among them, 49 were found in Yangtze River-Coilia, 31 in East China sea-Coilia, 30 in Chao Lake-Coilia, and 43 in Yellow River-Coilia. On comparing the compounds found in Coilia ectenes, we found that 20 volatile compounds were common to all four Coilia ectenes. Thirteen volatile compounds (propanal, 2-methyl-2-butenal, (E)-2-pentenal, 2-ethyl4-pentenal, 2-heptanone, 2,3-octanedione, 5-methyl2-heptanone, heptanol, (E)-2-octen-1-ol, 2-ethyl-4-methyl-1-pentanol, undecane, $N, N$-dimethyl-formamide, and $\mathrm{N}, \mathrm{N}$-diethyl-formamide) were found exclusively in Yangtze River-Coilia, two volatile compounds (2-butanone, 1-pentadecene) were found in Chao Lake-Coilia, and 9 volatile compounds (2,2-dimethyl-propanal, heptanal, 1H-pyrrole-2,5-dione, 5-ethyl-2 $(5 \mathrm{H})$-furanone, limonene, 2-ethyl-1H-pyrrole, 2-propyl-furan, 2-(2-pentenyl)furan, hexadecanoicacid, methyl ester) were found in Yellow River-Coilia.

A total of 35 odorants (including 33 volatile compounds and 2 unknown compounds) were identified in four Coilia ectenes by the olfactometric analysis (Table 2). Among all the odorants, there were 19 compounds in Yangtze River-Coilia, 21 compounds in East China sea-Coilia, 16 compounds in Chao Lake-Coilia and 20 compounds in Yellow River-Coilia. Based on the direct intensity method, assessors use a scale to measure the perceived intensity of a compound as it elutes, those odorants whose intensities were greater than or equal to 3 could be regarded as important odorants. Results showed that the important odorants including TMA (fishy, ammonia-like), 1-penten-3-ol (mushroom, cabbage), $\mathrm{N}, \mathrm{N}$-diethyl-formamide (roasted meaty), hexanal (grassy, earthy), (Z)-4-heptenal (fishy, boiled potato) and benzaldehyde (almond, metallic) in Yangtze River-Coilia; TMA, 1-penten-3-ol, ethylbenzene (nutty, floral), (Z)-4-heptenal, 1-octen-3-ol (mushroom, earthy) and nonanal (oily) in East China seaCoilia;TMA, 1-penten-3-ol, ethylbenzene, 1-octen-3-ol and nonanal in Chao Lake-Coilia, TMA, 1-penten-3-ol, hexanal, (Z)-4-heptenal,1-octen-3-ol and decanal (green, oily) in Yellow River-Coilia. 
Fig. 1 Total ion chromatograms of volatile compounds of four steamed Coilia ectenes a Yangtze river-Coilia, b East China sea-Coilia, c Chao lake-Coilia, d Yellow river-Coilia

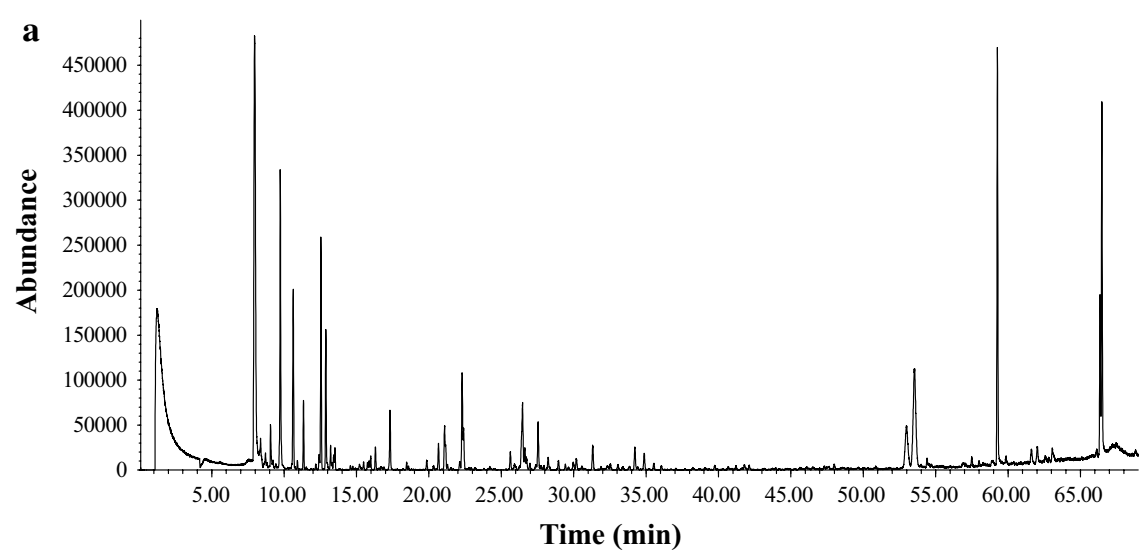

b

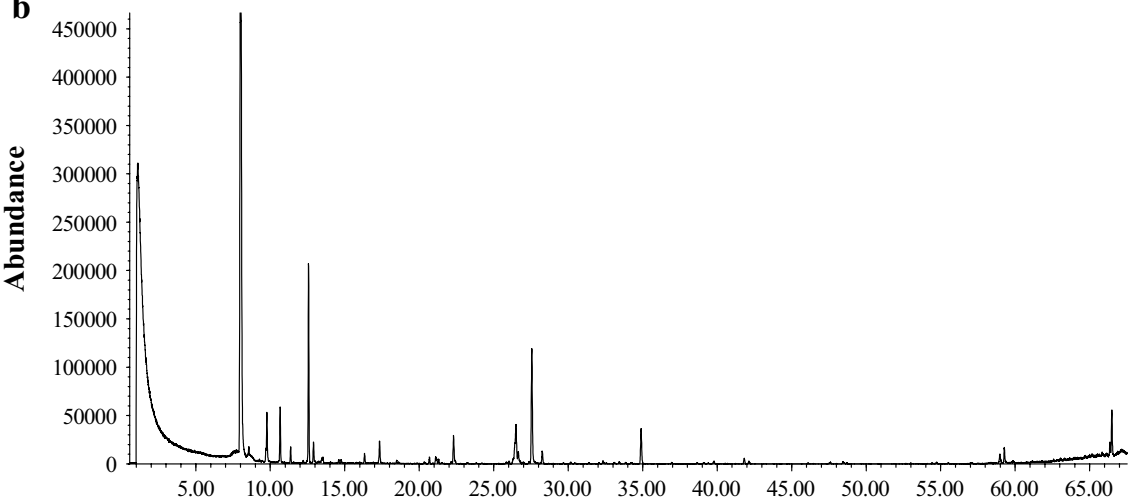

Time (min)

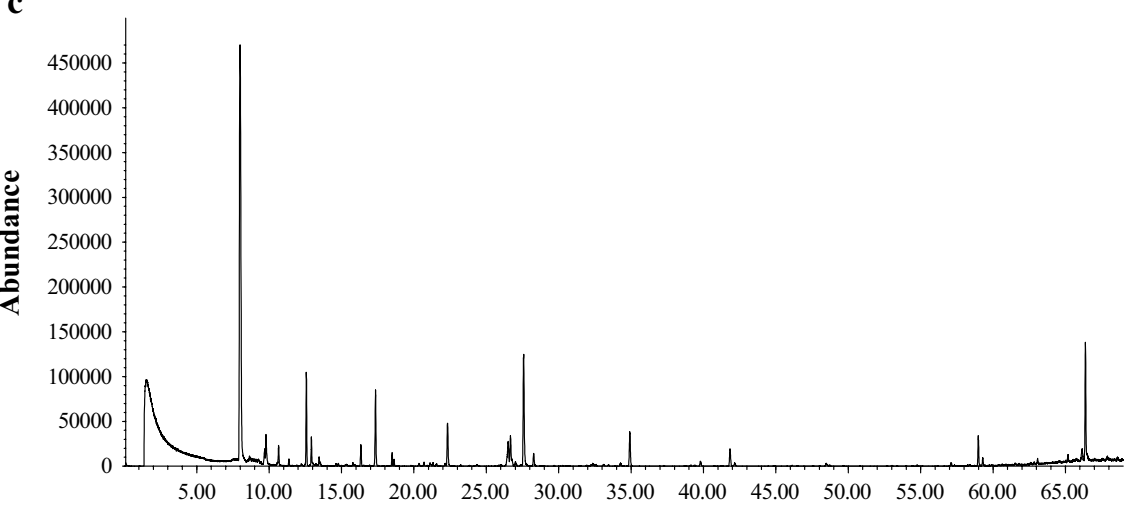

Time (min)

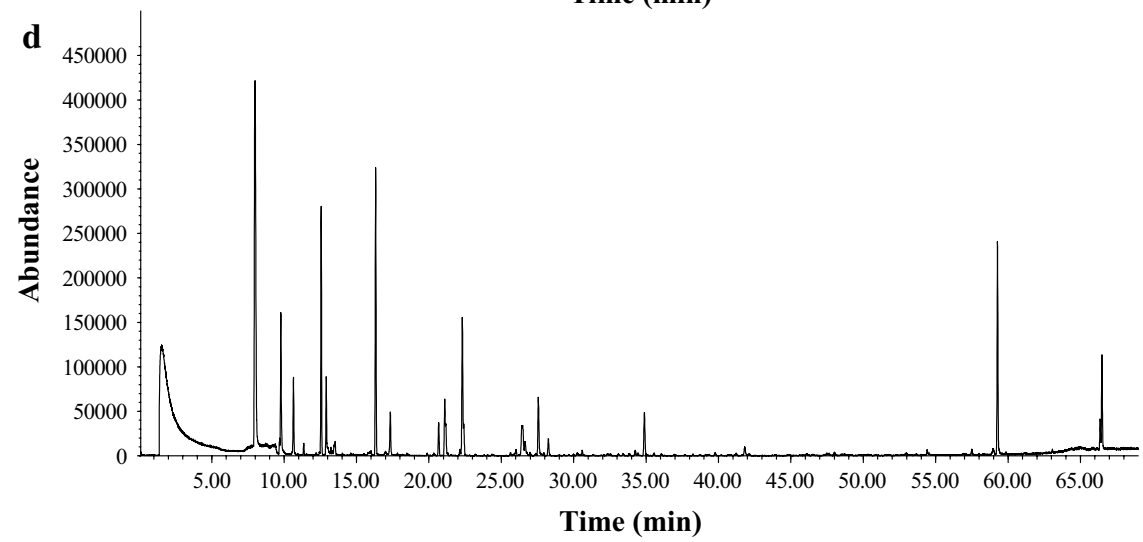


Table 1 Odor thresholds and peak area of volatile compounds identified in four steamed Coilia ectenes $(n=3)$

\begin{tabular}{|c|c|c|c|c|c|c|c|}
\hline \multirow[t]{2}{*}{$\mathrm{LRI}^{\mathrm{b}}$} & \multirow[t]{2}{*}{ Compounds } & \multirow[t]{2}{*}{ Identification $^{\mathrm{c}}$} & \multirow{2}{*}{$\begin{array}{l}\text { Odor threshold }(\mu \mathrm{g} / \\
\mathrm{kg})^{\mathrm{d}}\end{array}$} & \multicolumn{4}{|l|}{ Peak area $\left(10^{3}\right)$} \\
\hline & & & & A & B & $\mathrm{C}$ & $\mathrm{D}$ \\
\hline \multicolumn{8}{|c|}{ Aldehydes (19) } \\
\hline$<600$ & Propanal & MS & $0.69[1]$ & $17.44 \pm 2.02 \mathrm{a}$ & ND & ND & ND \\
\hline 657 & 3-Methylbutanal & MS, LRI & $1.6[2]$ & $8.77 \pm 1.69 \mathrm{a}$ & $3.24 \pm 1.04 b$ & $3.70 \pm 0.38 b$ & $4.79 \pm 0.35 b$ \\
\hline 697 & $\begin{array}{l}\text { 2,2-Dimethyl-pro- } \\
\text { panal }\end{array}$ & MS, LRI & NA & ND & ND & ND & $12.75 \pm 2.35 a$ \\
\hline 700 & 2-Methylbutanal & MS & $10[3]$ & $20.79 \pm 1.53 \mathrm{a}$ & $9.85 \pm 1.66 \mathrm{~b}$ & $9.92 \pm 1.77 b$ & ND \\
\hline 743 & 2-Methyl-2-butenal & MS, LRI & NA & $2.86 \pm 0.36 \mathrm{a}$ & ND & ND & ND \\
\hline 754 & (E)-2-Pentenal & MS & $0.98[4]$ & $9.28 \pm 1.43 \mathrm{a}$ & ND & ND & ND \\
\hline 802 & Hexanal $^{\mathrm{a}}$ & MS,LRI & NA & $91.99 \pm 6.82 b$ & $43.91 \pm 5.34 \mathrm{c}$ & $103.83 \pm 7.44 b$ & $115.07 \pm 12.11 \mathrm{a}$ \\
\hline 853 & (E)-2-Hexenal & MS, LRI & NA & $20.00 \pm 3.74 a$ & ND & $\mathrm{ND}$ & $13.75 \pm 1.41 \mathrm{~b}$ \\
\hline 895 & 2-Ethyl-4-pentenal & MS, LRI & NA & $15.07 \pm 3.34 \mathrm{a}$ & ND & ND & ND \\
\hline 901 & (Z)-4-Heptenal & MS, LRI & $3.4[5]$ & $21.58 \pm 2.21 \mathrm{a}$ & $2.98 \pm 0.54 b$ & ND & $24.05 \pm 4.25 \mathrm{a}$ \\
\hline 904 & Heptanal & MS, LRI & $6[6]$ & ND & ND & ND & $28.23 \pm 3.67 \mathrm{a}$ \\
\hline 975 & Benzaldehyde $^{\mathrm{a}}$ & MS, LRI & $10[7]$ & $165.95 \pm 28.22 \mathrm{a}$ & $116.10 \pm 6.15 b$ & $82.91 \pm 4.78 \mathrm{c}$ & $171.87 \pm 17.53 \mathrm{a}$ \\
\hline 1005 & Octanal $^{\mathrm{a}}$ & MS, LRI & $0.88[8]$ & $17.63 \pm 0.17 \mathrm{c}$ & $28.51 \pm 2.11 b$ & $27.34 \pm 3.12 b$ & $46.26 \pm 2.74 \mathrm{a}$ \\
\hline 1016 & $\begin{array}{l}\text { (E,E)-2,4-Heptadi- } \\
\text { enal }\end{array}$ & MS, LRI & $57[1]$ & $16.85 \pm 1.96 \mathrm{a}$ & ND & ND & $12.04 \pm 1.44 \mathrm{~b}$ \\
\hline 1084 & $\begin{array}{l}\text { 3-Methyl-benzalde- } \\
\text { hyde }\end{array}$ & MS, LRI & NA & $6.14 \pm 0.52 b$ & ND & $9.31 \pm 1.03 \mathrm{a}$ & $7.73 \pm 0.92 b$ \\
\hline 1107 & Nonanal $^{\mathrm{a}}$ & MS, LRI & $2[9]$ & $44.30 \pm 4.57 \mathrm{c}$ & $99.33 \pm 5.43 b$ & $92.29 \pm 2.93 b$ & $141.73 \pm 5.46 a$ \\
\hline 1179 & $\begin{array}{l}\text { 4-Ethyl-benzalde- } \\
\text { hyde }^{\text {a }}\end{array}$ & MS, LRI & NA & $7.60 \pm 1.14 c$ & $2.58 \pm 0.66 \mathrm{~d}$ & $11.83 \pm 1.09 b$ & $16.94 \pm 3.11 \mathrm{a}$ \\
\hline 1208 & Decanal $^{\mathrm{a}}$ & MS, LRI & $2.6[9]$ & $14.15 \pm 2.41 \mathrm{c}$ & $36.70 \pm 3.12 b$ & $38.07 \pm 3.27 b$ & $64.04 \pm 5.79 \mathrm{a}$ \\
\hline 1370 & 2-undecenal & MS, LRI & NA & ND & $18.51 \pm 3.89 b$ & ND & $21.04 \pm 2.45 \mathrm{a}$ \\
\hline \multicolumn{8}{|c|}{ Ketones (11) } \\
\hline$<600$ & 2-Butanone & MS, LRI & NA & ND & ND & $3.96 \pm 0.75 a$ & ND \\
\hline 693 & 2,3-Pentanedione & MS, LRI & NA & $31.88 \pm 4.39 a$ & $4.36 \pm 1.01 \mathrm{c}$ & ND & $16.22 \pm 2.14 b$ \\
\hline 696 & 3-Pentanone & MS & $0.06[10]$ & $7.95 \pm 1.46 \mathrm{a}$ & ND & ND & $7.86 \pm 0.73 a$ \\
\hline 825 & 3-Cyclohepten-1-one & MS, LRI & NA & $8.77 \pm 1.10 \mathrm{a}$ & $3.80 \pm 0.89 b$ & ND & $7.01 \pm 1.22 \mathrm{a}$ \\
\hline 888 & 2-Heptanone & MS, LRI & $62[11]$ & $4.08 \pm 1.17 \mathrm{a}$ & ND & ND & ND \\
\hline 980 & 2,3-Octanedione & MS, LRI & NA & $30.20 \pm 4.21 \mathrm{a}$ & ND & ND & ND \\
\hline 984 & 1H-Pyrrole-2,5-dione & MS, LRI & NA & ND & ND & ND & $8.64 \pm 1.33 a$ \\
\hline 1038 & $\begin{array}{l}\text { 5-Ethyl-2(5H)- } \\
\text { furanone }\end{array}$ & MS, LRI & NA & ND & ND & ND & $9.53 \pm 2.03 \mathrm{a}$ \\
\hline 1079 & Acetophenone & MS, LRI & NA & $9.18 \pm 1.57 \mathrm{a}$ & ND & $2.47 \pm 0.35 \mathrm{c}$ & $8.03 \pm 1.13 \mathrm{a}$ \\
\hline 1091 & $\begin{array}{l}\text { 5-Methyl-2-hep- } \\
\text { tanone }\end{array}$ & MS, LRI & NA & $5.94 \pm 1.82 \mathrm{a}$ & ND & ND & ND \\
\hline 1098 & $\begin{array}{l}\text { (E,E)-3,5-Octadien- } \\
\text { 2-one }\end{array}$ & MS, LRI & NA & $34.45 \pm 5.76 a$ & ND & $6.99 \pm 1.23 c$ & $21.98 \pm 2.38 b$ \\
\hline \multicolumn{8}{|c|}{ Alcohols (11) } \\
\hline$<600$ & 1-Propanethiol & MS & NA & ND & $19.00 \pm 2.13 b$ & ND & $23.39 \pm 3.24 \mathrm{a}$ \\
\hline 681 & 1-Penten-3-ol ${ }^{\mathrm{a}}$ & MS, LRI & $4300[1]$ & $196.10 \pm 4.22 b$ & $33.15 \pm 3.72 \mathrm{c}$ & $43.91 \pm 5.31 \mathrm{c}$ & $239.51 \pm 13.78 \mathrm{a}$ \\
\hline 735 & 2-Methyl-1-butanol & MS & $0.33[10]$ & $7.22 \pm 1.95 \mathrm{a}$ & $7.53 \pm 1.22 \mathrm{a}$ & $6.48 \pm 1.13 b$ & ND \\
\hline 761 & 1-Pentanol ${ }^{\mathrm{a}}$ & MS, LRI & $360[9]$ & $10.76 \pm 1.57 \mathrm{a}$ & $7.04 \pm 0.57 b$ & $5.33 \pm 0.86 c$ & $7.07 \pm 0.23 b$ \\
\hline 765 & (Z)-2-Penten-1-ol & MS & NA & $12.52 \pm 1.86 \mathrm{a}$ & $\mathrm{ND}$ & ND & $8.82 \pm 1.28 b$ \\
\hline 825 & (E)-3-Octen-2-ol & MS, LRI & NA & $2.73 \pm 1.28 b$ & ND & $30.58 \pm 3.45 a$ & ND \\
\hline 863 & 1-Hexanol & MS & $1.61[4]$ & $9.20 \pm 0.14 \mathrm{a}$ & $2.65 \pm 0.68 \mathrm{c}$ & $6.93 \pm 0.61 b$ & ND \\
\hline 966 & Heptanol & MS & $0.45[10]$ & $18.26 \pm 6.49 \mathrm{a}$ & ND & ND & ND \\
\hline 978 & 1-Octen-3-ol ${ }^{\mathrm{a}}$ & MS, LRI & $48[1]$ & $43.05 \pm 4.13 b$ & $19.23 \pm 2.34 c$ & $63.10 \pm 4.21 \mathrm{a}$ & $49.79 \pm 4.75 b$ \\
\hline
\end{tabular}


Table 1 continued

\begin{tabular}{|c|c|c|c|c|c|c|c|}
\hline \multirow[t]{2}{*}{$\mathrm{LRI}^{\mathrm{b}}$} & \multirow[t]{2}{*}{ Compounds } & \multirow[t]{2}{*}{ Identification $^{\mathrm{c}}$} & \multirow{2}{*}{$\begin{array}{l}\text { Odor threshold }(\mu \mathrm{g} / \\
\mathrm{kg})^{\mathrm{d}}\end{array}$} & \multicolumn{4}{|l|}{ Peak area $\left(10^{3}\right)$} \\
\hline & & & & $\mathrm{A}$ & $\mathrm{B}$ & $\mathrm{C}$ & $\mathrm{D}$ \\
\hline 1000 & (E)-2-Octen-1-ol & MS & $0.04[12]$ & $8.24 \pm 2.27 \mathrm{a}$ & ND & ND & ND \\
\hline 1025 & $\begin{array}{l}\text { 2-Ethyl-4-methyl- } \\
\text { 1-pentanol }\end{array}$ & MS, LRI & NA & $8.39 \pm 1.10 \mathrm{a}$ & ND & ND & ND \\
\hline \multicolumn{8}{|c|}{ Aromatics (8) } \\
\hline 669 & Benzene $^{a}$ & MS, LRI & $1500[13]$ & $329.21 \pm 31.58 \mathrm{a}$ & $233.28 \pm 12.65 c$ & $192.86 \pm 16.35 c$ & $276.95 \pm 25.33 b$ \\
\hline 776 & Toluene $^{\mathrm{a}}$ & MS, LRI & 1300 [9] & $44.74 \pm 1.81 \mathrm{~b}$ & $17.55 \pm 2.42 \mathrm{c}$ & $57.33 \pm 3.22 b$ & $87.10 \pm 11.34 \mathrm{a}$ \\
\hline 870 & Ethylbenzene $^{\mathrm{a}}$ & MS, LRI & $26[8]$ & $58.05 \pm 8.71 b$ & $11.89 \pm 1.43 c$ & $7.62 \pm 1.23 \mathrm{c}$ & $112.12 \pm 6.91 \mathrm{a}$ \\
\hline 879 & $p$-Xylene $\mathrm{e}^{\mathrm{a}}$ & MS & $1[14]$ & $38.33 \pm 8.71 \mathrm{a}$ & $22.24 \pm 1.76 b$ & $8.18 \pm 0.48 c$ & $6.53 \pm 1.21 \mathrm{c}$ \\
\hline 904 & Styrene $^{\mathrm{a}}$ & MS & $0.05[14]$ & $223.92 \pm 50.31 b$ & $46.80 \pm 4.23 d$ & $140.94 \pm 6.35 \mathrm{c}$ & $370.70 \pm 24.67 \mathrm{a}$ \\
\hline 972 & Phenol & MS & $58.59[15]$ & ND & $10.43 \pm 1.31 \mathrm{a}$ & $6.40 \pm 0.32 b$ & ND \\
\hline 1041 & Limonene & MS & $0.2[4]$ & ND & ND & ND & $18.84 \pm 1.45 \mathrm{a}$ \\
\hline 1214 & Naphthalene $^{\mathrm{a}}$ & MS, LRI & $40[16]$ & $9.51 \pm 0.33 b$ & $5.05 \pm 0.78 \mathrm{c}$ & $7.67 \pm 1.23 b$ & $14.20 \pm 1.25 \mathrm{a}$ \\
\hline \multicolumn{8}{|c|}{ Hydrocarbons (5) } \\
\hline 1400 & Undecane & MS & $10[14]$ & $15.83 \pm 4.84 \mathrm{a}$ & ND & ND & ND \\
\hline 1494 & 1-pentadecene & MS & $3.6[17]$ & ND & ND & $127.78 \pm 3.54 \mathrm{a}$ & ND \\
\hline 1500 & Pentadecane $^{\mathrm{a}}$ & MS, LRI & NA & $719.16 \pm 77.22 b$ & $161.56 \pm 6.23 c$ & $60.57 \pm 4.19 \mathrm{c}$ & $1621.18 \pm 23.76 \mathrm{a}$ \\
\hline 1600 & Heptadecane $^{a}$ & MS, LRI & NA & $215.82 \pm 34.85 b$ & $68.63 \pm 3.55 c$ & $263.79 \pm 25.66 b$ & $333.33 \pm 21.15 \mathrm{a}$ \\
\hline 1703 & $\begin{array}{l}\text { 2,6,10,14-tetrame- } \\
\text { thyl-pentadecane }\end{array}$ & MS, LRI & NA & $517.70 \pm 44.60 \mathrm{~b}$ & $304.21 \pm 7.89 b$ & ND & $1353.51 \pm 34.77 \mathrm{a}$ \\
\hline \multicolumn{8}{|c|}{$N$ - or $S$ - containing aromatic compounds (6) } \\
\hline$<600$ & Trimethylamine $^{\mathrm{a}}$ & MS, LRI & $2.5[18]$ & $1563.21 \pm 67.06 \mathrm{~d}$ & $4066.05 \pm 55.24 b$ & $2172.03 \pm 42.58 \mathrm{c}$ & $4665.83 \pm 59.36 \mathrm{a}$ \\
\hline 785 & $\begin{array}{l}N, N \text {-Dimethyl-for- } \\
\text { mamide }\end{array}$ & MS, LRI & NA & $9.54 \pm 2.27 \mathrm{a}$ & ND & ND & ND \\
\hline 921 & 2-Ethyl-1H-pyrrole & MS, LRI & NA & ND & ND & ND & $8.14 \pm 0.78 \mathrm{a}$ \\
\hline 936 & $\begin{array}{l}N, N \text {-Diethyl-forma- } \\
\text { mide }\end{array}$ & MS, LRI & NA & $5.23 \pm 1.60 \mathrm{a}$ & ND & ND & ND \\
\hline 991 & Dimethyl trisulfide & MS, LRI & NA & $11.43 \pm 0.75 \mathrm{a}$ & $3.60 \pm 0.34 b$ & ND & $10.63 \pm 1.78 \mathrm{a}$ \\
\hline 1588 & $\begin{array}{l}\text { Hexadecanoicacid, } \\
\text { methyl ester }\end{array}$ & MS & NA & ND & ND & ND & $11.71 \pm 1.35 \mathrm{a}$ \\
\hline \multicolumn{8}{|c|}{ Furans (3) } \\
\hline 702 & 2-Ethylfuran ${ }^{\mathrm{a}}$ & MS, LRI & NA & $23.67 \pm 5.61 b$ & $5.71 \pm 1.02 \mathrm{c}$ & $4.59 \pm 0.89 c$ & $30.52 \pm 2.54 \mathrm{a}$ \\
\hline 1001 & 2-Propylfuran & MS & NA & ND & ND & ND & $10.25 \pm 0.88 \mathrm{a}$ \\
\hline 1002 & 2-(2-Pentenyl)furan & MS, LRI & NA & ND & ND & ND & $7.12 \pm 1.67 \mathrm{a}$ \\
\hline Total & & & 49 & 31 & 30 & 43 & \\
\hline
\end{tabular}

Different letters in a row represent significant differences $(p<0.05)$

$N D$ not detected

NA Not available

$A$ Yangtze river-Coilia $B$ East China sea-Coilia $C$ Chao lake-Coilia and $D$ Yellow river-Coilia

${ }^{a}$ Volatile compounds identified in all four steamed Coilia ectenes

${ }^{\mathrm{b}}$ Linear retention index (LRI) on a DB-5MS column

c Means of identification: $M S$ mass spectra (identified from the mass spectra deposited in a database); LRI linear retention index (compared with the LRI in the literature)

d Odor thresholds were cited from the following 25 studies in the literature, all using air as the matrix: [Hall and Andersson 44], Bedborough and Trott [45], Bartschat and Mosandl [46], Tamura and others [47], Von Ranson and others [48], Deadman and Prigg [49], Khiari and others [50], Cometto-Muñizand Abraham [51], Nagata [52], Schnabel and others [53], Cain and others [54], Pyysalo and Suihko [55], Nauš [56], Zoeteman and others [57], Giri and others [58], Sävenhed and others [59], Tamura and others [60], and Amoore [61] 
Table 2 Odorants (Odor intensity $>1$ ) in four steamed Coilia ectenes by $\mathrm{GC}-\mathrm{O}$ detection $(n=3)$

\begin{tabular}{|c|c|c|c|c|c|c|c|}
\hline \multirow[t]{2}{*}{ LRI } & \multirow[t]{2}{*}{ Compounds } & \multirow[t]{2}{*}{ Identification } & \multirow[t]{2}{*}{ Odor description $^{\mathrm{a}}$} & \multicolumn{4}{|c|}{ Odor intensity $^{\mathrm{b}}$} \\
\hline & & & & $\mathrm{A}$ & $\mathrm{B}$ & $\mathrm{C}$ & $\mathrm{D}$ \\
\hline$<600$ & Trimethylamine $^{c}$ & MS, LRI, Odor & Fishy, ammonia-like & 4 & 4 & 4 & 4 \\
\hline$<600$ & 1-Propanethiol & MS, Odor & Sulfurous & - & 2 & - & 1 \\
\hline$<600$ & Propanal & MS, Odor & Green, fruity & 1 & - & - & - \\
\hline$<600$ & 2-Butanone & MS, LRI, Odor & Roast & - & - & 2 & - \\
\hline 657 & 3-Methylbutanal ${ }^{\mathrm{c}}$ & MS, LRI, Odor & Dark chocolate & 2 & 1 & 1 & 2 \\
\hline 669 & Benzene & MS, LRI, Odor & Floral & - & 2 & - & - \\
\hline 681 & 1-Penten-3-ol ${ }^{\mathrm{c}}$ & MS, LRI, Odor & Mushroom, cabbage & 3 & 3 & 4 & 3 \\
\hline 693 & 2,3-Pentanedione & MS, LRI, Odor & Roasted meaty, caramel & 2 & - & - & 1 \\
\hline 700 & 2-Methylbutanal & MS, LRI, Odor & Nutty, sweet & 1 & - & - & - \\
\hline 702 & 2-Ethylfuran & MS, LRI, Odor & Sweet corn & - & 2 & - & - \\
\hline 735 & 2-Methyl-1-butanol & MS, Odor & Fatty & - & 1 & 1 & - \\
\hline 754 & (E)-2-Pentenal & MS, Odor & Marine, fishy & 2 & - & - & - \\
\hline 761 & 1-Pentanol & MS, LRI, Odor & Floral & - & - & 1 & - \\
\hline 765 & (Z)-2-Penten-1-ol & MS, Odor & Mushroom, cabbage & - & - & - & 2 \\
\hline 776 & Toluene & MS, LRI, Odor & Painty, dusty & - & - & 1 & 2 \\
\hline 785 & $\mathrm{~N}, \mathrm{~N}$-Diethyl-formamide & MS, LRI, Odor & Roasted meaty & 3 & - & - & - \\
\hline 802 & Hexanal $^{c}$ & MS, LRI, Odor & Grassy, earthy & 3 & 2 & 2 & 3 \\
\hline 825 & (E)-3-Octen-2-ol & MS, LRI, Odor & Mushroom & - & - & 2 & - \\
\hline 825 & 3-Cyclohepten-1-one & MS, LRI, Odor & Roasted meaty, caramel & 2 & 2 & - & 1 \\
\hline 853 & (E)-2-Hexenal & MS, LRI, Odor & Green, fishy & 2 & - & - & - \\
\hline 870 & Ethylbenzene & MS, LRI, Odor & Nutty, floral & - & 3 & 3 & 2 \\
\hline 901 & (Z)-4-Heptenal & MS, LRI, Odor & Fishy, boiled potato & 3 & 3 & - & 4 \\
\hline 904 & Heptanal & MS, LRI, Odor & Green & - & 2 & - & - \\
\hline 975 & Benzaldehyde $^{c}$ & MS, LRI, Odor & Almond, metallic & 3 & 2 & 1 & 2 \\
\hline 978 & 1-Octen-3-ol ${ }^{\mathrm{c}}$ & MS, LRI, Odor & Mushroom, earthy & 1 & 3 & 3 & 3 \\
\hline 991 & Dimethyl trisulfide & MS, LRI, Odor & Cabbage, sulfurous & 2 & 1 & - & 2 \\
\hline 1005 & Octanal & MS, LRI, Odor & Grassy, oily & - & 1 & 2 & 1 \\
\hline 1016 & 2,4-Heptadienal & MS, LRI, Odor & Green, fishy & 2 & - & - & - \\
\hline 1079 & Acetophenone & MS, LRI, Odor & Nutty & - & - & 2 & - \\
\hline 1098 & (E,E)-3,5-Octadien-2-one & MS, LRI, Odor & Sweety, roasty & 1 & - & - & 1 \\
\hline 1107 & Nonanal & MS, LRI, Odor & Oily & - & 3 & 3 & 2 \\
\hline 1163 & Unknow & - & Corn, fruity & 2 & 1 & - & - \\
\hline 1179 & 4-Ethyl-benzaldehyde & MS, LRI, Odor & Marine & - & 1 & - & 1 \\
\hline 1208 & Decanal $^{\mathrm{c}}$ & MS, LRI, Odor & Green, oily & 2 & 2 & 2 & 3 \\
\hline 1300 & Unknow & - & Garlic, toasted meaty & - & 1 & - & 1 \\
\hline
\end{tabular}

$A$ Yangtze river-Coilia $B$ East China sea-CoiliaC Chao lake-Coilia $D$ Yellow river-Coilia

- Date not detected or available

a Odor description described by three experienced assessors during $\mathrm{GC}-\mathrm{O}$ detection

b Odor intensity reported by three experienced assessors during GC-O detection $(1$ = weak intensity, $2=$ moderate intensity, $3=$ strong intensity, and $4=$ very strong intensity)

${ }^{c}$ Odorants identified in all four steamed Coilia ectenes

\section{Discussion}

\section{Volatile compounds in four steamed Coilia ectenes}

A total of 19 aldehydes were found in four Coilia ectens. The 3-methyl-butanal and 2-methyl-butanal mainly contribute to the nutty almond notes, and may be formed by the Strecker degradation of leucine in the course of the Maillard reaction [13]. This reaction is known to be enhanced at high temperatures [14]. Benzaldehyde is a predominant compound in Yangtze River-Coilia, East China sea-Coilia and Chao Lake-Coilia, which originated from 
the oxidation of carbon-carbon double bonds in styrene and have been detected in fresh-baked sockeye salmon [15], roasted peanuts [16], and algae [17]. There are high levels of hexanal and nonanal in Chao Lake-Coilia. Hexanal, generally imparting a grassy odor, originates from the degradation of $n-6$ PUFA oxides and could be used as a degradation indicator of seafood products and meat from terrestrial animals [18]. Nonanal, imparting grassy and oily odors, is the predominant constituent in the autoxidation of linoleic acid [19]. Octanal and decanal may contribute to the more desirable aromas as well as the rancid odors and flavors that appear during the spoilage of fat and fatty foods [20]. Unsaturated straight chain aldehydes mainly contribute to the grassy, fatty, and fishy odors. For example, (Z)4-heptenal is characterized by a fatty and creamy odor, and this compound may be formed by a retro-aldol condensation of 2,6-nonadienal, which has been shown to be a major odor impact compound in a number of fresh marine creatures [21]. Other saturated aliphatic aldehydes such as octanal and decanal have grassy and oily odors.

Ketones, which are derived from PUFA oxidative degradation [22], have a higher threshold value compared to aldehydes and a relative small contribution to fish aroma $[23,24]$. However, the flavor differences among fish were mainly attributed to carbonyl compounds, so ketones have certain influences on flavor development [25]. Yangtze River-Coilia, Chao Lake-Coilia and Yellow River-Coilia have high levels of (E, E)-3, 5-octadien-2-one, which resulted from PUFA auto-oxidation and imparting sweet and roasted odors [4]. The compound 2, 3-pentanedione is an indicator of lipid oxidation in chilled fish muscle [26]. The 2, 3-pentanedione may contribute to roasted meat, caramel, and buttery odors, especially in cooked fillet of European catfish [27]. The compound 3-cyclohepten-1-one presents a high concentration in East China sea-Coilia, and has native and intense aromas that might be associated with the high fat content of East China sea-Coilia.

Alcohols are divided into two main classes: saturated alcohols and unsaturated alcohols. Saturated alcohols have higher flavor threshold values than unsaturated alcohols, therefore the contributions to fish odors is small $[28,29]$. Unsaturated alcohols have been identified as the major volatile alcohols in shellfish and cooked alligator meat [30] as well as many other aquatic products. Eleven alcohols were detected in Coilia ectenes; three alcohols (1-penten-3-ol, 1-pentanol, and 1-octen-3-ol) were common to all four species. The compound 1-penten-3-ol is the most abundant alcohol in Yangtze River-Coilia and Yellow River-Coilia, which was generated from PUFA oxidation [31]. It has been reported that 1-penten-3-ol presented in fresh whitefish at high concentration [32]. The compound 1-octen3-ol presents in Chao Lake-Coilia at high concentration, imparting desirable mushroom-like odors, mainly derived from the hydrogen peroxide degradation of linoleic acid [33].

Eight aromatics were identified in Coilia ectenes and six aromatics (benzene, toluene, ethylbenzene, $p$-xylene, styrene, and naphthalene) were common in all four species. Among them, toluene and $p$-xylene are generally believed to originate from environmental pollutants [34], which contributing to unpleasant off-flavors.

Hydrocarbons do not have any odor activities due to their high threshold values [35]. Nevertheless, intermediate alkanes, alkenes, arenes, and a small fraction of heterocyclic compounds may enhance the overall fish flavor [36].

The $N$ - and $S$-containing aromatic compounds, resulted from Maillard reactions between amino acids and reducing sugars, pyrolysis of amino acids (such as proline) and thiamine, dicarbonyl compounds from Maillard intermediate products, and degradations of thiamine via aldol condensation and aldehyde amine polymerization [31]. The $S$-containing aromatic compounds originate from the thermal degradation of methionine and cysteine [37], imparting meaty, maotai, and onion garlic flavors [31]. Even though some differences in the types and levels of $N$ - and $S$-containing aromatic compounds in Coilia ectenes, TMA $(53.82 \%)$ was present in all four species. TMA is generally believed to be produced by microbial metabolism in the presence of the precursor TMA oxide (TMAO) [38]. The presence of high levels of TMA in seafoods is undesirable, while TMA may add a pleasant crustacean-like odor at low levels [39].

Furans are a family of substances that play an important role in the flavor formation of Coilia ectenes, three furans have been identified in this study. The 1st odorant is 2-ethyl-furan, which was detected in all four Coilia ectenes, having a sweet corn odor. It was reported that 2-ethyl-furan is the oxidative degradation products of linolenic acid and commonly in crab [40-42]. The other furans, which were only detected in Yellow River-Coilia in this study, have also been reported in crab [43].

Above all, the main compounds associated with fish flavor are C6-C9 olefine aldehydes, enols and olefine ketones. There are more aldehydes, ketones, and alcohols in Yangtze River-Coilia and Yellow River-Coilia than in East China sea-Coilia and Chao Lake-Coilia. It is possible that Yangtze River-Coilia $(15.7 \pm 1.2 \%)$ and Yellow River-Coilia $(18.1 \pm 0.5 \%)$ have a higher fat content than East China sea-Coilia $(13.6 \pm 2.6 \%)$ and Chao LakeCoilia $(8.6 \pm 1.0 \%)$, aldehydes, ketones, and alcohols are produced from the oxidation and degradation of lipids. Additionally, environmental factors, e.g., water quality, sediment, algae and microbial species, may have significant effects on the flavor of Coilia ectenes [17]. The aromatic and hydrocarbon levels are similar to the four Coilia 
ectenes, other flavor compounds (e.g., TMA, dimethyl trisulfide compounds, and alkyl furans) are abundant in Yangtze River-Coilia and Yellow River-Coilia.

\section{Important odorants in four steamed Coilia ectenes}

A total of 35 odorants were identified in four Coilia ectenes (Table 2). Most of the odors are present at low levels but have important functions in the flavor development of Coilia ectenes. The characteristic flavors may originate from lipids, mainly short chain aldehydes and ketones [62]. Even though odorants are different among the four species, the main odor characteristics are fishy, grassy, mushroom, fatty, oily and meaty, which are consistent with a previous report [11]. TMA (fishy, ammonia-like), 1-penten-3-ol (mushroom, cabbage), $\mathrm{N}, \mathrm{N}$-dimethyl-formamide (roasted meaty), hexanal (grassy, earthy), ehtylbenzene (nutty, floral), (Z)-4-heptenal (fishy, boiled potato), benzaldehyde (almond, metallic), 1-octen-3-ol (mushroom, earthy), nonanal (oily), and decanal (green, oily) were the important odorants in four Coilia ectenes.

TMA levels are inversely proportional to fish quality [39]. TMA was perceived by the assessors to have average odor intensity values of 4 in four Coilia ectenes, showing the fishy and ammonia-like odors.

Unsaturated alcohols have low odor threshold values and with mushroom and metallic-like odors. The 1-penten3-ol and 1-octen-3-ol are commonly found in freshwater and seawater fish with strong odor activities [31]. The 1-penten-3-ol has an odor intensity value more than 3 in four Coilia ectenes, imparting mushroom and cabbage odors. The 1-octen-3-ol imparted the mushroom and earthy odors, mainly derived from the hydrogen peroxide degradation of linoleic acid [33], and has the lowest value in Yangtze River-Coilia.

Hexanal, contributed to the grassy, earthy scent of the four Coilia ectenes. It has been reported that hexanal mainly derived from the oxidation of linoleic acid and provides the "green" and fatty characters of fish and other seafoods [63]. Ethylbenzene, which has a nutty, floral odor, was previously identified with a relatively high intensity. The (Z)-4-heptenal, is a volatile flavor compound with low odor threshold value, which is characterized by a cooked fish, potato-like odor, might be derived from the water-mediated retro-aldol condensation of (E,Z)-2,6-nonadienal [64]. Chao Lake-Coilia has the highest levels of (E,Z)-2,6-nonadienal. Benzaldehyde, which was assigned an odor intensity of 3 in Yangtze River-Coilia, mainly contributed to the almond and metallic odors. It is derived from the oxidation of benzyl alcohol, which is catalyzed by dehydrogenases [65]. Decanal, which resulted from the oxidation of oleic and linoleic acids [11], has the higher intensity in Yellow River-Coilia than the other three Coilia ectenes, imparting unpleasant green and oily odors.

The compound 2, 3-pentanedione contributes to roasted meaty and caramel odors and buttery and caramel odors in salmon and trout [35]. The (E, E)-3, 5-octadien-2-one, which has a sweet, roast odor, has previously been detected in several seafood products [43].

In addition to known odorants, two unknown odorants were detected by the assessors. The first unknown odorant was detected as having a corn, fruity odor. The second unknown odorant had a garlic, roasted meaty odor. Although these odors could be detected by the assessors, they could not be identified by MS due to their weak signals. Nevertheless, even with weak signals and low estimated concentrations, these compounds may impart significant aromas in steamed Coilia ectenes, and should be evaluated in further studies.

Acknowledgments Research was supported by the Shanghai Engineering Research Center of aquatic-Product Processing and Preservation (11DZ2280300) and the Shanghai Municipal Natural Science Foundation (Grant No. 14ZR1420100). Thanks to the panelists who spent a good deal of precious time in describing the odor-active compounds in the steamed meat of Coilia ectenes.

Open Access This article is distributed under the terms of the Creative Commons Attribution 4.0 International License (http://creativecommons.org/licenses/by/4.0/), which permits unrestricted use, distribution, and reproduction in any medium, provided you give appropriate credit to the original author(s) and the source, provide a link to the Creative Commons license, and indicate if changes were made.

\section{References}

1. Liu Y, Wu Z, Hu M (2008) Advances on tapertail anchovy Coilia ectenes in China. Fish Sci 27:205-209 (in Chinese)

2. Xu G, Gu R, Zhang C et al (2009) Comparison and evaluation of nutrient composition of two ecological groups of Japanese grenadier anchovy-river-anchovy and sea-anchovy. Mar Fish 31:401-409 (in Chinese)

3. Jang HJ, Son HH, Lee DS (2011) Optimization of disk sorptive extraction based on monolithic material for the determination of aroma compounds from Lantana camara L. by gas chromatography-mass spectrometry. Bull Korean Chem Soc 32:4275-4280

4. Wei W, Sai-qi G, Ning-ping T (2013) Volatile flavor profiles of cooked Coilia ectenes Jordan meat. Sci Technol Food Ind 34:163-166 (in Chinese)

5. Van Ruth SM (2001) Methods for gas chromatography-olfactometry: a review. Biomol Eng 17:121-128

6. Linssen PH, Janssens GM, Roozen JP et al (1993) Combined gas chromatography and sniffing port analysis of volatile compounds of mineral water packed in polyethylene laminated packages. Food Chem 46:367-371

7. Plutowska B, Wardencki W (2008) Application of gas chromatography-olfactometry $(\mathrm{GC}-\mathrm{O})$ in analysis and quality assessment of alcoholic beverages: a review. Food Chem 107:449-463

8. Le Guen S, Prost C, Demaimay M (2000) Critical comparison of three olfactometric methods for the identification of the most potent odorants in cooked mussels. J Agric Food Chem 48:1307-1314 
9. Niimi J, Leus M, Silcock P et al (2010) Characterisation of odour active volatile compounds of New Zealand sea urchin roe using gas chromatography-olfactometry-finger span cross modality (GC-O-FSCM) method. Food Chem 121:601-607

10. Selli S, Rannou C, Prost C et al (2006) Characterization of aroma-active compounds in rainbow trout eliciting an off-odor. $\mathrm{J}$ Agric Food Chem 54:9496-9502

11. Wu W, Tao NP, Gu SQ (2014) Characterization of the key odor-active compounds in steamed meat of Coilia ectenes from Yangtze River by GC-MS-O. Eur Food Res Technol 238:237-245

12. Pollien P, Ott A, Montigon F et al (1997) Hyphenated headspacegas chromatography-sniffing technique: screening of impact odorants and quantitative aromagram comparisons. J Agric Food Chem 45:2630-2637

13. Iglesias J, Gallardo JM, Medina I (2010) Determination of carbonyl compounds in fish species samples with solid-phase microextraction with on-fibre derivatization. Food Chem 123:771-778

14. Aro T, Tahvonen R, Koskinen L et al (2003) Volatile compounds of Baltic herring analysed by dynamic headspace sampling-gas chromatography-mass spectrometry. Eur Food Res Technol 216:483-488

15. Nordvi B, Langsrud Ø, Egelandsdal B et al (2007) Characterization of volatile compounds in a fermented and dried fish product during cold storage. J Food Sci 72:S373-S380

16. Mason ME, Johnson B, Hamming MC (1967) Volatile components of roasted peanuts. Major monocarbonyls and some noncarbonyl components. J Agric Food Chem 15:66-73

17. Sugisawa H, Nakamura K, Tamura H (1990) The aroma profile of the volatiles in marine green algae. Food Rev Int 6:573-589

18. Prost C, Hallier A, Cardinal M et al (2004) Effect of storage time on raw sardine flavor and aroma quality. J Food Sci 69:S198-S204

19. Shahidi F (1994) Flavor of meat and meat products. Blackie Academic and Professional, London, pp 295-298

20. Forss DA (1973) Odor and flavor compounds from lipids. Prog Chem Fats Other Lipids 13:177-258

21. Chung HY, Cadwallader KR (1994) Aroma extract dilution analysis of blue crab claw meat volatiles. J Agric Food Chem 42:2867-2870

22. Cha YJ, Cadwallader KR, Baek HH (1993) Volatile flavor components in snow crab cooker effluent and effluent concentrate. $\mathrm{J}$ Food Sci 58:525-530

23. Prost C, Serot T, Demaimay M (1998) Identification of the most potent odorants in wild and farmed cooked turbot. J Agric Food Chem 46:3214-3219

24. Siek TJ, Albin IA, Sather LA et al (1971) Comparison of flavor thresholds of aliphatic lactones with those of fatty acids, esters, aldehydes, alcohols, and ketones. J Dairy Sci 54:1-4

25. Gasser U, Grosch W (1990) Primary odorants of chicken broth. Z Lebensm-Unters und Forsch 190:3-8

26. Iglesias J, Medina I (2008) Solid-phase microextraction method for the determination of volatile compounds associated to oxidation of fish muscle. J Chromatogr A 1192:9-16

27. Chung HY, Yeung CW, Kim JS et al (2007) Static headspace analysis-olfactometry (SHA-O) of odor impact components in salted-dried white herring. Food Chem 104:842-851

28. Wurzenberger M, Grosch W (1984) Stereochemistry of the cleavage of the 10-hydroperoxide isomer of linoleic acid to 1-octen-3-ol by a hydroperoxide lyase from mushrooms. Biochim Biophys Acta 795:163-165

29. Varlet V, Knockaert C, Prost C et al (2006) Comparison of odoractive volatile compounds of fresh and smoked salmon. J Agric Food Chem 54:3391-3401
30. Baek HH, Cadwallader KR (1997) Aroma volatiles in cooked alligator meat. J Food Sci 62:321-325

31. Cayhan GG, Selli S (2010) Characterization of the key aroma compounds in cooked grey mullet by application of aroma extract dilution analysis. J Agric Food Chem 59:654-659

32. Maarse H (1991) Volatile compounds in foods and beverages (eds) Chap.1. Marcel Dekker Inc, New York, pp 126-127

33. Josephson DB, Lindsay RC, Stuiber DA (1983) Identification of compounds characterizing the aroma of fresh whitefish. J Agric Food Chem 31:326-330

34. González S, Flick GJ, O'keefe SF et al (2006) Composition of farmed and wild yellow perch. J Food Compos Anal 19:720-726

35. Josephson DB, Lindsay RC, Stuiber DA et al (1987) Enzymic hydroperoxide initiated effects in fresh fish. J Food Sci 52:596-600

36. Sérot T, Regost C, Arzel J (2002) Identification of odour-active compounds in muscle of brown trout as affected by dietary lipid sources. J Sci Food Agr 82:636-643

37. Liu Y, Chen H, Sun B (2009) Recent advances in extraction and analysis of volatile compounds in fish. Food Sci 30:447-451 (in Chinese)

38. Unemoto T, Hayashi M, Miyaki K et al (1966) Formation of trimethylamine from DL-carnitine by Serratia marcescens. Biochim Biophys Acta 121:220-222

39. Cadwallader KR, Tan Q, Chen F et al (1995) Evaluation of the aroma of cooked spiny lobster tail meat by aroma extract dilution analysis. J Agric Food Chem 43:2432-2437

40. Chung HY (1999) Volatile components in crabmeats of Charybdis feriatus. J Agric Food Chem 47:2280-2287

41. Chen D, Zhang M (2006) Analysis of volatile compounds in Chinese mitten crab. J Food Drug Anal 14:297-303

42. Cha Y, Cho W, Jeong E (2006) Comparison of volatile flavor compounds in meat of the blue crab using V-SDE and SPME methods. J Korean Fish Soc 39:441-446 (in Korean)

43. Gu SQ, Wang XC, Tao NP et al (2013) Characterization of volatile compounds in different edible parts of steamed Chinese mitten crab. Food Res Int 54:81-92

44. Hall G, Andersson J (1983) Volatile fat oxidation products. I. Determination of odour thresholds and odour intensity functions by dynamic olfactometry. Lebensm Wiss Technol 16:354-361

45. Bedborough DR, Trott PE (1979) The sensory measurement of odours by dynamic dilution. Warren Spring Laboratory, Report Nr.: LR 299 (AP). Cited in Central Inst. for Nutrition and Food Research TNO, Zeist, Holland, Report Nr: A 84.220/090070

46. Bartschat D, Mosandl A (1998) Stereoisomeric flavour compounds LXXX: alkyl-branched alkanals-stereodifferentiation, structure elucidation and structure-function relationship. Z Lebensm Unters F A 206:165-168

47. Tamura H, Boonbumrung S, Yoshizawa $\mathrm{T}$ et al (2001) The volatile constituents in the peel and pulp of a green thai mango, Khieo Sawoei Cultivar. Food Sci Technol Res 7:72-77

48. Von Ranson C, Schnabel KO, Belitz HD (1992) Untersuchungen zur Struktur-Aktivitätsbeziehung bei Geruchsstoffen. IV: Struktur und Geruchsqualität bei aliphatischen alicyclischen und aromatischen aldehyden. Z Lebensm-Unters und Forsch 195:527-535

49. Deadman KA, Prigg JA (1959) The odour and odorization of gas. Research communication GC59. The Gas Council, London, p Code E2

50. Khiari D, Brenner L, Burlingame GA et al (1992) Sensory gas chromatography for evaluation of taste and odor events in drinking water. Water Sci Technol 25:97-104

51. Cometto-Muñiz JE, Abraham MH (2010) Odor detection by humans of lineal aliphatic aldehydes and helional as gauged by dose-response functions. Chem Senses 35:289-299 
52. Nagata Y (2003) Measurement of odor threshold by triangle odor bag method. Odor measurement review. Japan Ministry of the Environment, Government of Japan, pp 118-127

53. Schnabel KO, Belitz HD, Von Ranson C et al (1988) Untersuchungen zur Struktur-Aktivitäts-Beziehung bei Geruchsstoffen 1. Mitteilung: Wahrnehmungsschwellenwerte und Geruchsqualitäten von gesättigten aliphatischen und alicyclischen Verbindungen mit Sauerstoff-Funktion. Z Lebensm-Unters und Forsch 187:215-223

54. Cain WS, Wijk RA, Nordin S et al (2008) Independence of odor quality and absolute sensitivity in a study of aging. Chemosens Percept 1:24-33

55. Pyysalo H, Suihko M (1976) Odour characterization and threshold values of some volatile compounds in fresh mushrooms. Lebensm Wiss Technol 9:371-373

56. Nauš A (1975) Olphactoric properties of industrial matters. Charles University, Prague, pp 108-112

57. Zoeteman BCJ, Kraayeveld AJA, Piet GJ (1971) Oil pollution and drinking water odour. Water 4:367-371

58. Giri A, Osako K, Ohshima T (2010) Identification and characterisation of headspace volatiles of fish miso, a Japanese fish meat based fermented paste, with special emphasis on effect of fish species and meat washing. Food Chem 120:621-631
59. Sävenhed R, Borén H, Grimvall A (1985) Stripping analysis and chromatographic sniffing for the source identification of odorous compounds in drinking water. J Chromatogr A 328:219-231

60. Tamura H, Nakamoto H, Yang RH et al (1995) Characteristic aroma compounds in green algae volatiles. J Jpn Soc Food Sci 42:887-891 (in Japanese)

61. Amoore JE (1977) Specific anosmia and the concept of primary odors. Chem Senses 2:267-281

62. Hsieh RJ, Kinsella JE (1989) Lipoxygenase generation of specific volatile flavor carbonyl compounds in fish tissues. J Agric Food Chem 37:279-286

63. Frankel EN, Hu ML, Tappel AL (1989) Rapid headspace gas chromatography of hexanal as a measure of lipid peroxidation in biological samples. Lipids 24:976-981

64. Hallier A, Prost C, Serot T (2005) Influence of rearing conditions on the volatile compounds of cooked fillets of Silurus glanis. J Agric Food Chem 53:7204-7211

65. Abend AM, Chung L, Bibart RT et al (2004) Concerning the stability of benzyl alcohol: formation of benzaldehyde dibenzyl acetal under aerobic conditions. J Pharmaceut Biomed 34:957-962 